\title{
Rumänische Realität surrealistisch verkörpert: Joachim Wittstocks Erzählung Hades, (m)eine postmoderne Lesart
}

\author{
Carmen Elisabeth PUCHIANU \\ Prof. Dr., Transilvania-Universität, Kronstadt/Brasov. \\ E-Mail: carmenelisabethp@yahoo.de
}

\begin{abstract}
Our analysis of Joachim Wittstock's narrative entitled Hades and published thirteen years after the fall of the communist regime in Romania aims at pointing out the intimate connection between socio-political reality and personal experience reflected by the creative process of turning reality into fiction by writing. We consider the chosen narrative both as a political and literary statement, reflecting much of the way of life in Romania during the late 1980s. The narrative may be considered as some kind of withheld fiction and a pertinent comment of the author as to the role of fiction in a totalitarian regime.
\end{abstract}

Keywords: Joachim Wittstock, political reality, fiction

Der knapp sechzehneinhalb Seiten lange Text, dem wir uns hier widmen wollen, wurde 2002 im Erzählungsband Scherenschnitt ${ }^{1}$ erstveröffentlicht. Er hält sich wie sein Autor, Joachim Wittstock diskret zurück und fällt dem Leser zunächst im besten Fall durch den ungewöhnlichen Titel auf: Hades. Der Erzählband ist mit „Beschreibungen, Phantasien, Auskünfte“ untertitelt, und der Text könnte auf den ersten Blick jeder dieser Erzählkategorien zugeordnet werden. Diesen Eindruck bestätigt bereits der erste Absatz, der wie ein musikalischer Auftakt eine plötzliche Bewegung hervorruft und in einer additiven Reihe ausdrucksstarker

Vgl. Wittstock, Joachim: Hades. In: Scherenschnitt. Hermannstadt: Hora, S. 116-131. 
und überaus bewegter Bilder den ebenso chaotischen wie geordneten Ansturm von Reisenden auf eine Fähre vorführt:

Kaum hatte das Fährschiff „Per Scorillo“ angelegt, wurde es von all den Wartenden gestürmt. Über den leicht schwankenden Steg eilten Frauen und Männer hinüber, Kinder folgten ihnen. Kühnere Leute kletterten über die Brüstung. Habseligkeiten wurden ihnen nachgereicht, Taschen und Säcke, auch Koffer und Wasserbehälter, Melonen und Körbe mit Tomaten und Gurken. Alsbald war das wenig geräumige Schiff besetzt. ${ }^{2}$

Die konkreten Hinweise auf ein Fährschiff noch dazu mit dem Namen Per Scorillo oder, wie man an einer späteren Stelle des Textes erfährt, Decebalus per Scorillo, wecken vielfache Assoziationen, die für die Entschlüsselung des Textes wichtig sind, insofern sie sowohl auf eine bestimmte Topographie als auch auf eine genaue Chronographie hinweisen. Auch lässt sich hier bereits annehmen, dass der Text auf mehr als nur einer Diskursebene gründet und dass sich darin Privates, Persönliches mit Allgemeinem, Öffentlichem zu einem abgerundeten Artefakt verbindet.

Tatsächlich liegt dem Text eine persönliche Erfahrung des Schriftstellers, die er während wiederholter Sommerurlaubsaufenthalte an der Schwarzmeerküste gemacht hat, zugrunde:

Die Dobrudscha, die Untere Donau und das Donaudelta, die Schwarzmeerküste haben mich seit je angezogen [ ] Nicht von Konstanza und den südlich davon angeordneten Küstenstreifen und Badeorten soll im Folgenden die Rede sein, sondern von der weniger leicht zugänglichen Wasserplatte im Norden der Hafenstadt, mit ihren Flussarmen, Seen und Inseln.

Im August 1964 waren wir zu viert zu einer Exkursion ins Delta aufgebrochen [...]. Wir reisten zunächst per Eisenbahn und Bus, dann aber, von Tulcea aus, auf dem Wasserweg. Der mittlere Donauarm, Braţul Sulina, trug unser Motorschiff an Maliuc vorbei bis Crişan. Von dort ging's mit dem Schlepper nach

2 Ebd. S. 116. 
Caraorman und weiter in den Süden, wobei auch Fischerkähne zum Transport eingesetzt wurden. Wir passierten kleinere Kanäle und gelangten auf dem südlichen Donauarm, Braţul Sfântu Gheorghe, bis zur Meeresküste.[...] Unter Bekannten hatte es sich herumgesprochen, zu Strandaufenthalten in kaum berührter Natur reize die Landzunge, welche die Meeresküste von den ausgedehnten Seen südlich des Deltas trenne. Kein Badeortgewimmel habe man dort zu befürchten, bloß etliche Hütten und eine Fischerei umfasse die Portiţa genannte Anlegestelle für kleinere Motorschiffe; man zelte unweit des Meeresufers und verpflege sich selbst (gängigste Speise: Fisch). [...]. Drei Jahre hindurch steuerten wir das Ferienziel Portitza an (1978, 1979 und 1981). ${ }^{3}$

Wittstocks Text lässt sich ohne Weiteres aus der Perspektive privaten Erlebens lesen, sozusagen als literarische Auskunft wiederholter Ferienerlebnisse an der Meeresküste, zumal so gut wie jeder Leser diese Erfahrung selbst wenigstens ein Mal gemacht hat. Darüber hinaus bietet diese Dimension Anlass zu

3 Wittstock an Puchianu, 5.10.2018. Bis heute gilt Jurilovca als Geheimtipp für Urlauber, die die Abgeschiedenheit und Wildheit der, ,aquatischen Natur" bevorzugen. Die Beschreibung, die man im Internet lesen kann, hat einiges mit Wittstocks oben zitierten Zeilen gemeinsam, man betont die Unzugänglichkeit des Ortes, sein Auffinden ist eher das Ergebnis zufälliger Fügung: Jurilovca. Este o localitate amplasată în partea central-sudică a judeţului Tulcea, la aproximativ $89 \mathrm{~km}$ de Constanţa. Aici puteţi vizita Cetatea Argamum, situată la Capul Dolosman. Constituie unul dintre siturile arheologice cele mai interesante ale Dobrogei. Împreună cu insula Bisericuţa, aflată la câţiva kilometri în faţa sa, în apele lacului Razelm, ar putea constitui - şi datorită ineditului peisajului care le incadrează - unul din punctele de atracţie ale turismului nord-dobrogean. Accesul dificil deocamdată, atât pe uscat căt şi pe apă, au transformat acest punct, integrat odinioară drumurilor comerciale ce legau bazinul pontic de cel mediteranean, într-un loc izolat, în care vizitatorii ajung de cele mai multe ori accidental, rămănând fascinaţi de frumuseţea peisajului. (Vgl. http://transporturi.transevren.ro/excursii_detalii.php?ruta=constanta_portitei, am 20/9/2018). 
weit mehr als nur dem Festhalten literarischer Ferienauskünfte und tritt dabei hinter der sachlich objektiven Haltung des Erzählers zunehmend in den Hintergrund. Es sind hauptsächlich rhetorische Passagen, die eine direkte Assoziation zu der Figur des Schriftstellers ermöglichen, Andeutungen seiner Gegenwart ganz im Stil von Hitchcocks Momentauftritten in seinen Filmen, wie etwa der Hinweis auf den einzigen Passagier, der ein Büchlein aufgeschlagen hat, den Lesenden, der sich Gedanken macht während der Überfahrt und dabei die Haltung eines aufmerksamen, etwas verschmitzt lächelnden, augenzwinkernden Beobachters einnimmt:

Sollten alle Fahrtgenossen eine Seele haben? Die Kinder eine unbewusste, noch unausgebildete, die Erwachsenen eine erfahrene, gar harmonisch gereifte Seele? Zeit war genug, beim gemächlichen Schlingern des Fahrzeugs die Gesichter zu betrachten, um festzustellen, was sich darin spiegele ... Befanden sich sämtliche Passagiere ihrer eigenen Seele gegenüber und damit großer Pein ausgesetzt? So zumindest war im Büchlein zu lesen, das einer der Touristen aufgeschlagen hatte. [...] Der Lesende ließ das kleine Buch auf seine Knie sinken. Sich gegenüber, grübelte er, indes sein Blick über die Reisegesellschaft glitt, das heißt wohl: ein Sich-Begegnen in unterschiedlich deutlicher Selbstbewusstheit, die Einen sehen da sicher klarer, die Anderen verstehen es weniger gut, sich gegenüberzutreten und zutreffend einzuschätzen. Oder ist Seelentätigkeit solcher Art nur einigen vorbehalten, vielleicht bloß einem Einzigen - dem Weltgeist, der sich spiegelgleich in der Wasseroberfläche verstofflicht hat? ${ }^{4}$

Die ironische Hinterfragung bestätigt, dass sich die private Ebene des Textes einer weiteren öffnet bzw. dass diese sich auf dem Hintergrund konkreter Gegebenheiten der tatsächlichen Lebensrealität ereignet. Im Verlauf der Erzählung wird sich die auktoriale Stimme zunehmend als allwissende, ironische und sachliche Erzählinstanz herausstellen.

Kehren wir aber noch einmal zum Auftakt der Erzählung zurück: Eine Menge Wartender werden beim Anlegen der Fähre

4 Wittstock, S. 117f. 
in frenetische Bewegung versetzt, man ist bemüht, möglichst schnell das Gefährt zu erklimmen um einen Platz zu ergattern, man kann sich denken, dass die Fähre wenig Platz für Passagiere bietet, sodass der Eindruck von chaotischem aber auch zwanghaft geordnetem Anstehen, Andrängen und Anstürmen entsteht. Wer wie Wittstock seine besten Jahre während der kommunistischen Zeit in Rumänien verbracht hat, kennt die Situation aus eigener Erfahrung. Die Unmenge an Gepäck, an Habseligkeiten, die mitgeschleppt werden, nebst den zu verladenen Kisten und Kanistern ergänzen das Bild und verleihen ihm groteske Züge. Die Überfahrt mit der Fähre Per Scorillo, von der man nicht weiß, wie lange sie dauern wird, verspricht alles andere als angenehm und bequem zu sein für die Reisefreudigen, zumal der Fährmann nicht gerade höflich oder zimperlich mit seinen Passagieren umgeht. Er scheint eine gewisse Autorität zu haben bzw. versteht er sich als Vertreter einer solchen, denn er erteilt den Leuten strenge, kurz angebundene Weisungen, ja sogar Befehle, denen stattgegeben werden muss, ist man dem Fährmann immerhin auf Gedeih und Verderb ausgeliefert, zumindest für die (ungewisse) Dauer der Überfahrt:

Mit barschen Worten vertrieb er die Passagiere aus dem zeltplachenbedeckten Teil des Fahrzeugs an die vor der Sonne ungeschützte Seite, dort sollten sie sich zusammenkauern. „Platz für die Ware!“‘

Unter Gemurre räumten die Gescholtenen das Feld. Bierfässer rollten heran, eine Wasserzisterne wurde herbeigeschleppt, Kisten schwang man auf die Plattform. Wer jetzt noch als Mitfahrer das Schiff besteigen wollte, wurde abgewiesen. ,Ausgeschlossen! Soll »Per Scorillo« wegen euch sinken? Fort von hier! “5

Das Urlaubmachen gehörte während des Kommunismus in das öffentliche Politprogramm der Partei und oblag einer offiziellen Vorschrift wie alles andere im kommunistischen Rumänien

5 Ebd. S. 116. 
auch. Jeder Werktätige hatte das Recht auf mindestens 15 und höchstens 24 bezahlte Urlaubstage im $\mathrm{Jahr}^{6}$, so hatte man mit etwas Glück und guten Beziehungen zum Beispiel die Möglichkeit über die Gewerkschaft einen zwölftägigen Aufenthalt an der Schwarzmeerküste oder an andern beliebten Kur- und Urlaubsorten in Rumänien für sich und die Familie zu verhältnismäßig günstigen Konditionen zu buchen, man wohnte dann sogar im Hotel und aß im Hotelrestaurant dreimal am Tag nach streng geregelter Programmierung, meistens in mehreren Schichten. Man bekam allerdings einfaches Kantinenessen vorgesetzt, im Gegensatz zu jenen, die sich den Luxus eines Aufenthalts über die rumänische Reiseagentur ONT Carpaţi leisten konnten, oder gar im Vergleich zu den ausländischen Gästen, die allerdings vor den Blicken der Einheimischen aus guten Gründen abgeschirmt wurden. Der Urlaub steht in den kommunistischen Zeiten im Grunde unter zweideutigem Vorzeichen: Er wird von oben angeordnet als notwendige Abwechslung vom Werkalltag und zur Regeneration der Arbeitskraft für neue Herausforderungen, bietet aber auf privater Ebene eine authentische Ausbruchsmöglichkeit aus den Zwängen des Systems in eine zeitweilige scheinbar unbeaufsichtigte Freiheit, ganz gleich ob man diese auf den Kämmen der Karpaten, an der Schwarzmeerküste oder im Donaudelta suchte. Diese Ambiguität empfindet Joachim Wittstock als Schriftsteller intensiv und verarbeitet sie in seiner Erzählung auf ganz besondere Weise. Zunächst verfasst er aber andere Texte, in denen die erwähnten Urlaubsorte Anlass zu literarischen Überlegungen bieten: das Gedicht Im Donaudelta ${ }^{7}$ und Portitza zu deutsch:

${ }^{6}$ Das Urlaubsgeld betrug im Durchschnitt etwa 1100 Lei, d.h. etwa bis $\mathrm{zu} 80 \%$ des Monatslohns. Vgl. Concedii și vacanțe în România comunistă-documentar.https://www.youtube.com/watch?v=vNeAfgMldCY, Zugriff am 16. 11. 2018.

7 Vgl. Wittstock: Botenpfeil. Gedichte. Cluj: Dacia, 1972, S. $66 \mathrm{f}$. 
die Kleine Pforte. ${ }^{8}$ In beiden Texten geht es unterschwellig um die metaphorische Analogie zwischen dem Erleben ,aquatischer Horizonte ${ }^{\text {" } 9}$ und dem uneingeschränkten Bedürfnis nach Freiheit. Später, genauer gesagt Anfang der Neunzigerjahre gesellt sich noch ein weiterer Text hinzu, und zwar Delta. Drei Donauarme, drei Diskurse ${ }^{10}$. Aus einem regelrechten Amalgam von Eindrücken und Erfahrungen entsteht schließlich der Text Hades, von dem Wittstock zusammenfassend und knapp informiert: ,,die Erzählung Hades stützt sich auf Aufzeichnungen, die ich im aquatischen Horizont, also vor Ort, um 1980 angefertigt hatte, sowie auf spätere Notate (manche gar nach $1990 \mathrm{zu}$ Papier gebracht) “". ${ }^{11}$

Wenn wir nun den Text weiterlesen, finden wir den ersten Eindruck bestätigt: Wir befinden uns atmosphärisch in den düstersten Momenten des rumänischen Kommunismus. Der mit der Fähre erreichte Urlaubsort entpuppt sich als ein mythisch geschichtsträchtiger Ort rumänischer Identität. Man nennt das Land nicht ohne Ironie Burebistum und knüpft dadurch an die Benennung des Fährschiffes, vornehmlich aber an die rumänische Geschichtsschreibung und an den Stellenwert der rumänischen Geschichte im Gesamtkontext der kommunistischen Diktatur an. Geschichte wird zur politischen und ideologischen Legitimierung des Machtsystems vereinnahmt, sie wird den ideologischen Bedürfnissen der Partei und ihres heroischen Führers entsprechend zurechtgemacht und neu geschrieben:

Pe măsură ce România lui Ceaușescu se îndepărta, se rupea de trecutul său, istoria era totmai intens valorizată și pusă la lucru.[...] Epoca ceaușistă a fost și o colosală (și în bună măsură reușită) manipulare prin istorie.[...] S-au definit și câteva trăsături fundamentale

8 Vgl. Wittstock: Parole Atlantis. Erzählende und betrachtende Prosa. Cluj-Napoca: Dacia, 1980, S. 95 f.

9 Wittstock an Puchianu, 5.10.2018.

${ }^{10}$ Vgl. Wittstock: Kurator, Söldner, Gouverneur und andere Prosa. Bukarest: Kriterion, 1998, S. 69-72.

${ }^{11}$ Wittstock an Puchianu, 5.10.2018. 
- și constante - ale istoriei românești. Acestea erau vechimea, unitatea, continuitatea și lupta pentru independență. ${ }^{12}$

Die großangelegten Inszenierungen mit geschichtlichem Hintergrund dienen mehr und mehr dazu, die Person des Staatspräsidenten als unumstrittenen Helden der rumänischen Geschichte im Kontext einer jahrhundertelangen Reihe von Heroen, die bis ins frühe Reich der Daker reichte, zu feiern. Dass man dabei in die Bereiche des Absurden geriet, spielt allein für die Literaten eine Rolle:

Paroxismul s-a atins prin comemorarea, în 1980, a 2050 de ani de la constituirea statului dac, ,centralizat și independent“ (evident!), sub conducerea lui Burebista. Un eveniment pur și simplu fabricat, [...] fiindcă nimeni nu știe de fapt când s-a constituit statul dac sau când a început domnia lui Burebista. Ceaușescu a vrut să fixeze bornele unei istorii mărețe: de la Burebista la el însuși. ${ }^{13}$

Wittstocks Text ist vor diesem Hintergrund zu lesen: Das aufgezwungene Geschichtsverständnis von eigenständiger rumänischerKulturund Tradition und dasBewusstsein desjahrhundertelangen Währens vor Ort finden literarische Verkörperung sowohl in der Ankunfts- als auch in der Besichtigungssequenz der Erzählung. Man landet nach der etwas ermüdenden weil eintönigen Fahrt mit der Schiffsfähre auf dem ,Schutzgebiet altererbter Volkskultur" ${ }^{\text {"14 }}$, das sich den Gästen bald als Grenzgebiet und Unterwelt erschließt, mitsamt seiner ganz besonderen Gesetzen, Vorschriften und Verboten. Wittstocks Burebistum erweist sich von Anfang an weniger als transitorischer Urlaubsort mit südländischem Flair, sondern viel eher als Ort streng überwachten und dauerhaften Bleibens und als solcher ist er für die manipulatorische Belehrung viel eher als für die

${ }^{12}$ Boia, Lucian: Strania istorie a comunismului românesc (și nefericitele ei consecințe), Bucuresti, 2016, S.157-158.

${ }^{13}$ Ebd. S. 161.

${ }^{14}$ Wittstock, Hades, S.121. 
Urlaubsunterhaltung geeignet. Fährmann und Soldaten treten im Namen einer höheren und offensichtlich gefürchteten, weil autoritären Macht auf, sie erteilen strenge Weisungen und sprechen Verbote aus, die allerdings nicht für sie gelten:

Auf dem Schiff, dessen vollständiger Name „Decebalus Per Scorillo“ lautete, war es still geworden, als die Passagiere von Grenzwächtern in Empfang genommen wurden. Diese trugen grasgrüne Uniformen und - obwohl Jenseitige, dem Hades zugehörig - sahen sie eigentlich wie Menschen aus. Einer der Grenzer verlangte den Passagieren die Akten ab.

Die Ankömmlinge dürstete es, sie wollten sich an dem mitgebrachten Getränk laben und drängten sich an die Wasserzisterne und an die Bierfässer heran. Soldaten vertrieben sie mit energisch akzentuierten Worten.

„Hier im Grenzgebiet ist alles verboten“, sagte einer der mit Pistole ausgerüsteten Unteroffiziere. ,Entfernt euch nicht!“”, befahl er routinemäßig, ,erst müssen die Papiere in Ordnung sein.“

Er goss sich in den bereitstehenden Becher Rotwein ein, und auch die anderen Uniformträger, gleich welchen Ranges, darunter Offiziere, nahmen gelegentlich einen Schluck Bier oder Wein zu sich, aus Gläsern, die in ihrer Nähe auf den Tischen und Bänken der Anlegestelle standen ... Alles war verboten, aber bei der Überwachung der Verbote war man hier, im Schattenreich, offenbar nicht sehr pedant. Und so war manches, wenn nicht fast alles erlaubt. ${ }^{15}$

Die Szene kann ohneweiters als rumäniendeutsches Pendant zu Orwells Losung 'Alle Tiere sind gleich. Aber manche sind gleicher' aus der Farm der Tiere aufgefasst werden. Es folgt in der Erzählung eine Episode, die wir Besichtigungsepisode nennen wollen und ebenfalls auf Authentischem beruht: Jurilovca hat, so Wittstock, ein Heimatmuseum aufzuweisen.

Dessen Schwerpunkt lag auf den Lipowanern, ihrer Geschichte und Kultur. Dem Volkstum nach waren die Lipowaner slawisch, der

${ }^{15}$ Ebd. S. 119-120. 
Konfession nach strenggläubig orthodox, allerdings mit Normen und Riten, die sie zur sektiererischen Gemeinschaft werden ließen. Religiöse Motive waren in dem Museum nicht besonders hervorgehoben, im Gegenteil - Laïsches dominierte, und die taktisch-spekulative Ausrichtung auf die sozialistische Ideologie und Obrigkeit war gar etwas aufdringlich. Hochbemüht waren die Ausführungen des Museumsleiters, eines alten Herren ukrainischen Herkommens, einst Offizier in der zaristischen Armee, der gleichsam Sprachrohr und Anwalt der Lipowaner geworden war. ${ }^{16}$

In der Erzählung wird der Museumswärter als Mentor bezeichnet, dem die Aufgabe zufällt, die Neuankömmlinge landeskundlich zu unterweisen und in die Geschichte des Ortes einzuführen, die er in seinem bescheidenen Heim sorgsam konserviert hat „in großer Achtung vor den Ahnen [...] dass man einen Eindruck von dem Menschenschlag dieses einzigartigen Landstrichs erhält"17. Wie in einem skurrilen Wachsfigurenkabinett wird hier Geschichte vorgeführt:

Als sich etwa dreißig Personen um ihn geschart hatten, sprach er sie an: „Liebe Leute! Touristen! Oder darf ich sie als Dauergäste von Burebistum ansprechen, unserer einzigartigen, sich erfreulich mehrenden Siedlung? Seien Sie uns herzlich willkommen! Folgen Sie mir bitte in mein bescheidenes Heim. In großer Achtung vor den Ahnen habe ich es so eingerichtet, dass man einen Eindruck von dem Menschenschlag dieses unvergleichlichen Landstrichs erhält.

Der Erste, der sich in unserem vielfach gesegneten Gebiet ansässig machte, hieß Lipow, und als solcher wurde er ins Bevölkerungsregister eingetragen. Weil der Zweite, der da kam, ihm ähnlich sah, mit niemals geschnittenem Bart und ungekürztem Haar, hat man auch ihn als »Lipow« verzeichnet, und die Nächsten von gleicher Art nannte man ebenfalls »Lipow« ..." [ ] Im ersten Raum saß einer aus dem Geschlecht der Lipows am Tisch und blickte in das vor ihm aufgeschlagene Buch. Ein grau-weißer Vollbart reichte auf seine Brust. Den Fischer und zugleich Landmann sah man ihm nicht auf den

${ }^{16}$ Wittstock an Puchianu, 5.10.2018.

${ }^{17}$ Wittstock, Hades, S. 123. 
ersten Blick an, indes ließen die schwer auf der hölzernen Tischplatte ruhenden Fäuste erkennen, dass er gewohnt war, körperliche Arbeit $\mathrm{zu}$ verrichten.

Das Buch hingegen offenbarte die geistige Ausrichtung seines Lebens. Wenn der Mentor auch nicht verriet, ob die in kyrillischen Lettern gehaltene Schrift die Bibel oder eine Predigtensammlung war, so machte er dennoch auf einen Umstand aufmerksam: Dies sei ein handgeschriebenes Buch, und derartigem gaben die Lipowaner den Vorzug, bis ins späte 19. Jahrhundert, weil sie im Buchdruck ein Teufelswerk sahen.

Der Mann am Tisch reagierte nicht auf den Zugang der Gruppe wie sollte er auch? Längst hatte man ja erkennen können, dass er nicht lebte, sondern einem Lebenden in voller Größe bloß nachgebildet und mit typischen Kleidern angezogen war, mit weitläufig geschnittener heller Bluse und dunklen Hosen. Oder sollte dies ein wirklicher Erdenbewohner sein, der, für sein Fortleben in der Unterwelt, nach allen Regeln der Konservierung präpariert worden war? ${ }^{18}$

Die rumänische Gegenwart der 1980er Jahre mit ihren politischen Konnotationen findet sich in diesem Panoptikum sorgsam konserviert und präpariert wieder, wofür auch Trikolore und das gerahmte Präsidentenporträt stehen: „Um gewichtigen Vorschriften zu genügen, war in einem Winkel des Raums eine Trikolore in einen Ständer gesteckt, und von der Wand blickte, aus einem Bilderrahmen, der Landespräsident auf das Publikum."19

Schon früher in der Erzählung spielt der Landespräsident eine Rolle: Auf dem Fährschiff werden die Passagiere aufgefordert, jemanden in Gedanken mitzuführen, sich jemandes zu erinnern zum eigenen Halt vielleicht in der neuen Umgebung, die sich bald als Unterwelt erweisen wird, oder als Möglichkeit andere in diese Welt hinüber zu denken - zu teleportieren. Auf

${ }^{18}$ Wittstock, S. 123-124

${ }^{19}$ Ebd. S. 124. 
die Frage, wer denn mitgeführt werden könne, ein Lebender oder gar ein Toter, ergeht folgende Antwort:

„Am Besten ist es“, tuschelte ein Mann mit seinem Nachbarn, und es war, als würden Galeerensklaven miteinander wispern, ,ich bringe unseren Landespräsidenten bei. Das ist für mich günstig, weil er der Höchste ist, und das heißt dann auch, er verschwindet, und wir sind ihn los.“"Wenn man ihn beibringt", flüsterte der zweite Galeerensklave, „dann auch seine Frau, die große Wissenschaftlerin. Übernimmst du ihn, dann will ich mich an sie erinnern. Und weg ist weg, ein für alle Mal." Sie lachten glucksend, auf Sklavenart, der Aufsicht gewärtig, bereit, ihre Mienen sogleich wieder in unterwürfigem Ernst erstarren zu lassen. ${ }^{20}$

Die unverblümte Anspielung auf den Landespräsidenten und dessen Frau, der „großen Wissenschaftlerin“ - in der offiziellen Titulatur der Zeit sogar von Weltruhm - bedarf an dieser Stelle keiner weiteren Erörterung. Burebistum entpuppt sich als Unterwelt und Ort der ständigen Beaufsichtigung, so wie Rumänien während der Achtzigerjahre seinerseits zu einem solchen Ort mutiert war, selbst wenn der ursprüngliche Impetus der Fährgäste dorthin zu reisen einer der Befreiung und Erholung gewesen ist; auch scheinen die Urlauber einer eigenartigen Metamorphose unterworfen worden zu sein: Sie sind Galeerensklaven, die von Grünuniformierten, die sich als Grenzwächter entpuppen und soldatisches Gebaren an den Tag legen, strengstens beaufsichtigt werden.

Diese untere Welt ist auch ein Ort der ,Sondierungen“21 und der Prospektionen, wie wir erfahren, „weil man Erdöl sucht" ${ }^{\text {"222 }}$, und damit wird dem Ort auf ironische Weise das Potential einer Unterwelt wiederum in Frage gestellt:

Der Präsident hat gesagt, wo Erdöl ist, kann von Unterwelt keine Rede sein. Und die große Wissenschaftlerin lässt sich laufend über

${ }^{20}$ Wittstock, Hades, S.118 -119.

${ }^{21}$ Ebd. S. 120.

${ }^{22}$ Ebd. S. 121. 
die Ergebnisse der Sondierungen berichten. Sie ist es ja gewesen, die vor mehreren Jahren darauf gedrungen hat, auf dem Meer die Bohrinsel Decebal zu errichten. Diesen Kurs geht sie unbeirrt weiter, weil sie glaubt, dass Schürfungen auch entlang der Küste Erfolg versprechen. Demnach stehen die Chancen schlecht für die Unterwelt $^{23}$

Der Text schließt mit einer ebenso bewegten Szene wie er eingesetzt hat: Man wird zum Sammelpunkt beordert, denn immerhin geht es darum, eine endgültige Aufnahme in dieser eigenartigen Welt zu erlangen und für längere, ja unabsehbar lange Zeit dort ansässig zu werden. Man bewegt sich einer Herde gleich einem Tor entgegen, das man passieren muss, um in die neue Welt hineinzugelangen, die sich als Welt mit eigenen Regeln herausstellt, ,an die sich jedermann genauestens halten muss. [...] Und nun das Besondere: Nach reiflicher Überlegung muss man sich entscheiden, was man zu tun wünscht, denn dabei bleibt es für lange Zeit. "24

„Zum Sammelpunkt“, hieß es, als die Letzten sich der Gesellschaft näherten, ,alle herkommen!“ Und da die „Per Scorillo“-Passagiere vollzählig waren, hieß es: „Zur Aufnahmestelle!“ Schon setzten sie sich in Bewegung.

Auf Anweisung des Fährmanns, der überraschenderweise wieder zugegen war, hielt man auf flachgewalzten Muschelresten auf eine etwa meterhohe Mauer zu, die aus übereinandergeschichteten, nur wenig durch Mörtel verbundenen Bruchsteinen bestand und sich quer über den Küstenstreifen erstreckte. Der Weg führte auf ein geräumiges Tor $\mathrm{zu}$, das diesseits und jenseits der eisengefassten Holzflügel mit einem breiten Dach versehen war, zum Schutz vor Regen oder-wie jetzt-vor den immernoch kräftigen Sonnenstrahlen des späten Nachmittags. Die Pforte öffnete sich und wurde von dem Konvoi durchschritten. ${ }^{25}$

${ }^{23}$ Ebd. 121.

${ }^{24}$ Ebd. 127-128.

${ }^{25}$ Wittstock, S. 126-127. 
Die Analogie zur rumänischen Gesellschaft während der kommunistischen Diktatur wird hier erneut deutlich durch die Idee des Spiels, der Unterhaltung und des Spektakels hervorgestrichen. Womit die Neuankömmlinge im gerade eben betretenen Burebistum oder Hades Daciae konfrontiert werden, entpuppt sich als merkwürdiger Rummelplatz, als eine Art Luna Park, wie er in den Jahren der Diktatur an der Meeresküste (und an anderen Orten) angelegt worden war. Zu dem befristeten Erholungsurlaub gehört das angeordnete Spektakel, das hemmungslose Geschrei und Getummel auf der halsbrecherischen Achterbahn oder auf dem Riesenrad zum Urlaub einfach dazu. Nach dem Motto Brot und Zirkus dem Volk, sollte man wenigstens den Zirkus haben, wenn das Brot schon rationiert ausgegeben wur$\mathrm{de}^{26}$. Man stürzte sich in kindisches Vergnügen, um die Grenzen der eigenen Kräfte, aber auch jene des Systems auszutesten. Im prunkvollen Spektakel politischer Kundgebungen und Großaufmärsche inszenierte sich die Macht am 1. Mai oder am 23. August, im Spiel und im Spektakel des Rummelplatzes konnte man die Macht hintergehen und sich selbst in einen Zustand der Freiheit katapultieren. Das Geschrei war Ausdruck von Angst und Befreiung zugleich. In Wittstocks Text nimmt die so erlebte rumänische Realität absurd surrealistische Dimensionen an, die sowohl mit Unterhaltung und Befreiung zu tun haben, als auch mit Angst und Beklemmung:

Man sieht ja wohl dort in der Ferne eine Schaukel, die einen Turner in die Höhe schnellen lässt, er schaukelt also nicht von einer Seite zur anderen, sondern von oben nach unten. Das hat der Betreffende

${ }^{26}$ Ceaușescu hatte bereits 1970 anlässlich seiner ersten USA-Reise den Unterhaltungspark Disneyland besichtigt und war so begeistert davon, dass er 1980 sogar ein Dekret erlassen haben soll, um in Bukarest einen analogen Park errichten zu lassen. Vgl. https://ceausescunicolae.wordpress.com/2011/08/09/ceausescu-a-vrut-sa-faca-disneylandin-parcului-tineretului-decret-dat-de-ceausescu-pe-22-august-1980/ (Zugriff am 28. 1. 2019). 
sich ausgewählt. Ebenfalls auf ihren eigenen Wunsch stehen andere als Betreuer daneben und sorgen dafür, dass der Springer nicht verunglückt.

Einer fragte: „Und der springt immerfort in die Luft, vielleicht einen ganzen Tag lang?“

Der Beamte sah ihn eine Weile prüfend und auch in nachsichtiger Überlegenheit an und antwortete: „Einen Tag? Nein, länger, viel länger, möglicherweise immer.“ Und er ergänzte: ,,»Immer « bedeutet hier allerdings etwas Anderes als drüben. So wie ja auch ein Tag eine ganz andere Dauer hat als jenseits der Mauer.“27

Aus der Nähe betrachtet wirkt der Rummelplatz tatsächlich wie ein Ort der Verdammnis. Die Neuankömmlinge und künftigen Bewohner der unteren, oder besser, der Unterwelt, Urlauber oder Galeerensklaven, wer weiß das schon so genau, sehen dem Treiben auf dem Rummelplatz zu, ihnen fehlt die Energie, einen Ausweg zu finden oder die Lage auf irgendeine Weise zu hinterfragen, dazu sind Hitze und Trägheit zu groß. Auch ist das Tor, das man passiert hat, verschlossen und kann oder darf nicht mehr geöffnet werden, die Zeit nimmt ihrerseits ungewohnte Ausmaße an. Die neue Seinsweise wird von dem Mann am besten veranschaulicht, ,der es verstand, in die Luft zu springen, und der sich dann fallen ließ, um erneut hochzufedern. "'28 Ein grotesker und nicht minder bedauernswerter Sisyphos, dessen Spektakel alle in den Bann zieht:

Schräg auseinanderstrebende Stangen aus leichtem Metall bildeten das Gerüst, von dessen oberen Enden Gummizüge hinabhingen, jeweils an zwei von insgesamt vier Fixpunkten befestigt. Die elastischen Seile, etwa fingerdick in der Stärke, mündeten in Riemzeug, das dem Springer um die Hüfte und auch die Schenkel geschlungen war. Um seinem Auf und Ab noch zusätzliche Elastizität zu geben, befand sich zu seinen Füßen eine geräumige Liege, die allein schon

27 Wittstock, S. 128.

${ }^{28}$ Ebd. S. 129-130. 
ausgereicht hätte, eine wippende Bewegung beträchtlicher Ausmaße in Gang zu bringen.

Da sah man also den Mann, der sich, in gleichmäßigen Schwingungen, etwa sechs Meter hoch wuchten konnte und der dann wieder absackte und - in endloser Folge - sich durch die Luft bewegte, jetzt und immerfort. $^{29}$

Die Unterwelt, mit der man hier konfrontiert ist, scheint kein Reich des Todes und der Toten zu sein, sondern eher eines des Lebens und Lebender unter zweideutigem Vorzeichen. Die agierenden Figuren erinnern an Figuren von Kafka, Orwell oder Ransmayer: Sie agieren am Rand der Welt, in einem ebenso historischen wie fiktiven Burebistum, das sich zu einer überzeitlichen Dystopie und Weltmetapher auszuweiten scheint.

\section{Anstelle eines Fazits}

Der Text bietet einem eine doppelte Perspektive: zum Einen spiegelt er die traumatisierende Wirklichkeit der rumänischen Diktatur auf dem Hintergrund scheinbar belangloser Urlaubsszenerie wieder, zum Andern verbirgt er einen komplizierten und langwierigen Prozess des literarischen Schöpfungsaktes. Der Text verdeutlich dem Leser von heute, dass ein Autor zwar immer auf die konkreten Gegebenheiten der Realität reagiert, indem er eine Position bezieht, findet jedoch die Literarisierung nicht immer zeitgleich statt, sondern bedarf einer kürzeren oder längeren Gärungsphase. Letzteres ist der Fall von Joachim Wittstocks Erzählung Hades, von der man eigentlich hätte denken wollen, es handelte sich um einen sogenannten Schubladentext. Den Auskünften Joachim Wittstocks zufolge wurde der Text tatsächlich erst 2002 erstveröffentlicht, nicht etwa weil er ein Schubladentext gewesen wäre, sondern weil er in mehreren Anläufen und Schreibansätzen anhand vielfacher Eindrücke

${ }^{29}$ Wittstock, S.130. 
und Notate erst in den Neunzigerjahren zu Papier gebracht worden ist:

Die Erzählung ,Hades “ stützt sich auf Aufzeichnungen, die ich im aquatischen Horizont, also „vor Ort“", um 1980 angefertigt hatte, sowie auf spätere Notate (manche gar nach 1990 zu Papier gebracht). [ ]Es gab Ausführungen, die ursprünglich in den Zusammenhang zu passen schienen und sich in den Bericht über eine längere Seefahrt einbringen ließen. Ich nahm sie aber wieder heraus, modelte sie um und veröffentlichte sie separat ${ }^{30}[$ ] Will man genau sein, muss man sagen: Das Jurilovka-Portitza-Amalgam wird noch von einer weiteren Szenerie ergänzt, auf der sich die Vergnügungspark-Momente vom Schluss der Erzählung abspielen. Sie wurden von einem anderen Dobrudscha-Schauplatz auf die abgeschiedene, stille Gegend einer gedachten Unterwelt projiziert. ${ }^{31}$

Hades widerlegt ohne Wenn und Aber die Meinung, Joachim Wittstock sei ein unpolitischer Schriftsteller. Ganz im Gegenteil, er weist seinen Autor, der mit den Mitteln der Ironie und der Parodie aus der Sicht eines auktorialen Erzählers, der sich als Geist seiner Erzählung nicht ganz zurücknimmt, eine Welt offenbart, die einen in die Bereiche einer ebenso gelebten wie fiktionalisierten absurd surrealen Welt versetzt und das krasse Bild der rumänischen Realität während der Ceauşescu Ära darstellt als durchaus politisch aus.

Darüberhinaus zeichnet sich der Text von Joachim Wittstock durch eine bei weitem tiefere weil philosophische / weltanschauliche Dimension aus, sodass der Text über die konkrete sozio-politische Bedeutung hinaus aktuell bleibt. Dafür sorgt die Omnipräsenz des Erzählers, seine eigenartige Körperlichkeit und ironische Verfremdung der verkörperten Geschichte. Daraus resultiert eine weitere Komponente der Erzählung und zwar ihr performativer Charakter: Realität entstehen dadurch und sie verkörpern sich im Spannungsfeld von Spiel / Unterhaltung und

${ }^{30}$ Vgl. Wittstock: Dem Ende zu In: Scherenschnitt, 2002, S. 111-115.

${ }^{31}$ Wittstock an Puchianu, 5.10.2018. 
Manipulation. Auf diese Weise generiert der Text in seinen einzelnen Episoden fiktionale Realität und wandelt sie in eine Surrealität, die im Zeichen des Absurden und der allgemeinen Losigkeit $^{32}$ steht. Auch in diesem Sinn bleibt der Text über seine scheinbare Vordergründigkeit hinaus überzeitlich politisch aktuell.

\section{Literatur:}

\section{Primärliteratur}

Wittstock, Joachim: Hades. In Scherenschnitt, Hermannstadt, 2002, S.116-131.

Sekundärliteratur

Boia, Lucian: Strania istorie a comunismului românesc (și nefericitele ei consecințe), București, 2016

Puchianu, Carmen Elisabeth: Roter Strick und schwarze Folie. Postmoderne Theateradaptionen auf den Leib geschrieben, Transilvania Universitätsverlag, 2016

\section{Internetquellen}

Concedii și vacanțe în România comunistă - documentar. https://www.youtube.com/watch? $\mathrm{v}=\mathrm{vNe} A f g M l d C Y$, Zugriff am 16/11/2018.)

http://transporturi.transevren.ro/excursii_detalii. php?ruta $=$ constanta_portitei (Zugriff am 20.09.2018)

https:/ceausescunicolae.wordpress.com/2011/8/09/ceausescu-a-vrut-sa-faca-disneyland-in-parcului-tineretului-decret-dat-de-ceausescu-pe-22-august-1980/ (Zugriff am 28.1.2019)

Vgl. Puchianu, Carmen Elisabeth: Roter Strick und schwarze Folie. Postmoderne Theateradaptionen aufden Leib geschrieben, Transilvania Universitätsverlag, 2016, S. 71f. 